\title{
El dispositivo Badiou/ Beckett y el episodio aristofánico del "croar de las ranas"
}

fecha de recepción. fecha de aceptación.

\begin{abstract}
Resumen
El presente artículo hipotetiza las posibilidades comparatísticas que se establecen entre el episodio artistofánico conocido como "el croar de las ranas", hipotexto de la tradición comediográfica griega, y su revisitación tanto en Watt de Samuel Beckett (1906-1989) como Citrouilles de Alain Badiou como "acontecimiento-ruido". El episodio de Ranas (B⿱́㇒一 $\tau \rho \alpha \chi o$ ) de Aristófanes (444 a. C.-385 a. C.) aparece reescrito en ambos autores contemporáneos en torno al fénome de la nominación y la incomunicación. El interés de Badiou, en tanto filósofo y dramaturgo, es redefinir figuraciones de la inarticulación y conflictos entre el ser y el lenguaje en base a los postulados dramáticos de Beckett: la forma en que el autor irlandés reescribe en Watt el episodio del croar de las ranas y la manera en que el autor de $\mathrm{El}$ ser y el acontecimiento, gran lector y estudioso de Beckett, articula, en su propia producción dramatúrgica, las relaciones posibles entre Aristófanes y Beckett como autores dramáticos centrales en su propia experiencia y práctica dramatúrgica.
\end{abstract}

\section{The Badiou / Beckett device and the Aristophan episode of "croaking of frogs"}

\footnotetext{
Abstract

The present article hypothesizes about the comparative possibilities between the Aristophanic episode known as "the croaking of frogs", a hypotext in Greek comedic tradition, and its revisitation as a "noise-event" both in Watt by Samuel Beckett (19061989) and Citrouilles by Alain Badiou. The frog episode (Bátpaðoı) of Aristophanes (444-385 BC) is rewritten by both contemporary authors around the notions of nomination and lack of communication. Badiou's interest, both as a philosopher and as a playwright, is to redefine the figures of inarticulacy and the conflicts between being and language based on Beckett's dramatic postulates: the way in which the Irish author
}

Palabras clave

Croar nominación incomunicación acontecimiento sonoridad
Keywords

croaking nomination lack of communication event noise 
1. Véase el trabajo de Saba Akim (2013: 50-59).

2. Lecercle aporta una lectura de "doble vía" en sus esclarecedores "Badiou reads Beckett" y en "Beckett reads Badiou" en Lecercle (2010).

3. Recuérdese el íncipit de la novela y sus posibilidades de lectura desde la teoría de Badiou: “¿Dónde ahora? ¿Cuándo ahora? ¿Quién ahora? Sin preguntármelo. Decir yo. Sin pensarlo. Llamar a esto preguntas, hipótesis. Ir adelante,

Ilamar a esto ir, llamar a esto

adelante. Puede que un día, venga

el primer paso, simplemente haya permanecido, donde, en vez de salir, según una vieja costumbre, pasar días y noches lo más lejos posible de casa, lo que no era lejos. Esto pudo empezar así. No me haré más preguntas, Se cree sólo descansar, para actuar mejor después, o sin prejuicio, y he aquí que en muy poco tiempo se encuentra uno en la imposibilidad de hacer nada. Poco importa cómo se produjo eso. Eso, decir eso, sin saber qué. Quizá lo único que hice fue confirmar un viejo estado de cosas. Pero no hice nada. Parece que hablo, y no soy yo, que hablo de mí, y no es de mí. Estas pocas generalizaciones para empezar. ¿Cómo hacer, cómo voy a hacer, qué debo hacer, en la situación en que me hallo, cómo proceder?", en Beckett (1966: 35).

4. Sobre las cuestiones referidas al "poema latente" y a la prosa como textualidad genérica Artus-Bouvet así lo expone: “La prose désignerait en ce sens la capacité générique du poème, qui lui permet d'accueillir à la fois une duplicité énonciative, la motivation d'un récit et de ses simulacres, l'organisation d'un doute méthodique, et la profondeur d'une réflexivité de type métadiscoursif. (continúa en página 23)

5. Para otro enfoque de la relación entre sujeto y tiempo en Beckett véase el apartado

"Beckett, poeta del tiempo" en el artículo de la destacada teatróloga francesa Anne Ubersfeld en Pellettieri (2000: 265-271). rewrites in Watt the episode of the croaking of frogs and the way in which the author of Being and Event, a great reader and scholar of Beckett, articulates, in his own production, the possible relations between Aristophanes and Beckett as central dramatic authors in his own experience and practice.

Badiou declara conocer la obra de Samuel Beckett desde mediados de la década del 50, cuando él era un "sartreano consumado", que "con sospechas" se internaba en las primeras obras que el irlandés produce enteramente en lengua francesa, con el rótulo "caricatural" que ubicaba a Beckett entre los escritores del absurdo, ${ }^{1}$ la incomunicación y la desesperación: ${ }^{2}$

Incluso en la supuesta desesperación de Beckett existe el claro de una posibilidad inaudita. Ese teatro muestra que la situación, desde luego, es desesperante, pero que un Sujeto puede hacer que prevalezca su propia ley numerosa. Cuando una mujer semienterrada, cuyo marido impotente se arrastra detrás de ella sin dirigirle jamás la palabra, declara: ‘Qué lindo día era!’, hay que tomar lo que dice al pie de la letra y en lo más mínimo como una confusión irrisoria. (Badiou 2014:68)

La lectura de El innombrable revierte esa consideración ${ }^{3}$ y traslada los recelos en una fuerte indagación sobre la finitud a partir de un primer "desprendimiento" del pensamiento beckettiano: "fuera de la obstinación de las palabras no hay sino tinieblas y vacío" (BK, 10). No leído ya con guiños existencialistas, Beckett se le revelaba a Badiou como un escritor de la valentía y de la mesura en torno a esa “obstinación" medular por las palabras. La larga frecuentación de los escritos beckettianos - y su heterodoxia - significaron una prueba de valentía frente al hecho literario y frente a la 'excepción' en que esa obra se constituía por fuera de los géneros establecidos, ya que ningún género literario "conviene" — según Badiou - a la "apuesta beckettiana"; para Badiou toda la textualidad beckettiana está más bien "gobernada” por un "poema latente" —casi un retorno a Mallarmé ${ }^{4}$ - cuyo "timbre inaudito" se refuerza por una idea (persistente) de 'distancia': distancia de sí mismo, de la naturaleza y de la lengua materna. Badiou llamará a esta escisión una "fractura íntima" en el interior mismo de la frase beckettiana, que produce dos efectos 1) "aísla palabras para rectificarlas (...) por medio de añadidos de epítetos o de correcciones” y 2) por “repentinas expansiones líricas”, en donde el cálculo sonoro "apacigua la tensión del espíritu y lo envuelve en la nocturnidad de la reminiscencia" (Beckett, el infatigable, 14). Las dos operaciones que Badiou abstrae de Beckett intentan lograr un "movimiento prosódico unificado" que corresponda a la rectificación y a la expansión y sus derivados: declaración / declinación e interrupción / estiramiento. ${ }^{5}$

Por otra parte, de esa primera etapa de "ascesis metódica" en lengua francesa, Badiou reconoce tres funciones: 1) el movimiento y el reposo; 2) el ser y 3) el lenguaje: "Un "personaje" no deja de ser, en todo momento, aquel que dispone un trayecto, una identidad, un parloteo cruel" (Badiou 2007: 17).

Tomamos estas tres instancias como inspiradoras para el propósito de este artículo: analizar el ser de Ahmed en Citrouilles, cuarta pieza de la Tetralogía de Alain Badiou y su catábasis, en cuya busca de identidades en el "Infierno del teatro", mediante su "lengua de ingenio", reconocemos no sólo el famoso episodio del croar del coro de ranas en Ranas de Aristófanes, como impar hipotexto, sino también el reverbero de un episodio gráfico-musical de Watt de Samuel Beckett.

Badiou publicó en tanto dramaturgo publicó durante la década del 90 una tetralogía que tiene como protagonista al "personaje diagonal" de Ahmed y cuya culminación es 
la pieza Citrouilles, que revisita el tema y la estructura dramática de Ranas de Aristófanes. Citrouilles, como hace Ranas, pretende que el personaje marginal de Ahmed se introduzca en el infierno de ultratumba del teatro a los fines de rescatar con finalidades cívicas al mejor dramaturgo que pueda hacerse cargo de la crisis general de las sociedades postcapitalistas occidentales. Tal como Ranas presentaba una famosa contienda entre Esquilo y Eurípides, Citrouilles - haciendo una recorrida descomunal por la historia del teatro en occidente y todas las figuras claves fallecidas que se encuentran en ese Hades teatral - plantea una actualización del famoso agón final de la obra confrontando la dramaturgia del comunista Bertold Brecht con el católico Paul Claudel. Durante ese "descenso", tal como en Ranas ocurría con Dioniso, en Citrouilles Ahmed será quien, en medio de su delicado periplo, se verá conturbado ya no por "el ditirámbico de las ranas" y su metáfora batracia sino por calabazas y su metáfora vegetal como representantes inoperantes y obstaculizadores mediante los cuales Badiou parodiza - a través de duras y testarudas calabazas que representan a toscos programadores, autores, operadores, críticos y periodistas - las dificultades que estos energúmenos interponen al real desarrollo de la cultura y de la manifestación central de la civilidad: el teatro y su acceso masivo al pueblo.

Badiou - siguiendo sus propios términos "sustractivos", pero con refracciones derivadas del método de "reducción drástica" 6 o los "ejercicios de empeoramiento" de Beckett - pondrá en diálogo su propia dinámica constructiva de la obra y de los personajes de Citrouilles con las resoluciones dramáticas que lo han desde siempre inspirado del autor irlandés. Si el 'hablador' de El innombrable, encadenado a un gran macetero situado a la entrada de un restaurante, es sustraído a la movilidad (Badiou, 2017:19), Badiou tomará de Beckett, por medio de este procedimiento, ${ }^{7}$ una suerte de axiomática depurada aplicada a sus piezas y, en especial a Citrouilles, donde intenta concentrar las fuerzas entre ser y lenguaje.

Badiou utilizará una metáfora del todo teatral para indagar los espacios cerrados y abiertos que son esenciales en la postulación espacial ("el trabajo poético de localización") de Beckett al denominar "escena del ser" (o "la asignación del lugar del ser") a la fijación de un dispositivo ficcional "que busca captar en la lengua el tiempo de conversión del ser en nada” (Badiou 2007:25); pero, para el filósofo francés, la supina operación beckettiana - reflejada en la escritura - es concentrar ahí el fenómeno de la voz y su contraparte, su "parte de noche": el silencio. La voz prolifera o se abisma, se precipita o trastabilla ${ }^{8}$, y en ambos 'vectores' de manifestación, en esas 'formas del habla', siempre se vuelve "inevitable".

Ante estas dos instancias —el "lugar del ser" y el "rumiar de la voz" abriéndose camino ${ }^{10}$ - , Badiou considera que Beckett produce el advenimiento del "acontecimiento". En este punto la poética de Beckett y la relectura teatral de Badiou se concentran en, al menos, dos fenómenos clave: la noción de acontecimiento o del "aparecer" que, acaso, "cristaliza" en Godot como "promesa siempre vuelta a comenzar de su venida", y, en la "escena del dos" o "amor" (entendido como aquello de lo que son capaces un "verdugo" y una "víctima”) que Badiou observa en las "parejas” beckettianas.

A este último aspecto dedica Badiou la singularidad más grande del teatro de Beckett: la manera en que éste "restringe" o sustrae la teatralidad "a los efectos posibles del dos". Vemos en este dos (o en estas parejas) formas de releer la relación entre amo y esclavo o entre los personajes contrarios del agón final de Citrouilles - y por ende de Ranas de Aristófanes- como modos de "captura verbal de todas las consecuencias de la dualidad", según destaca Badiou.

Cobrará interés para nuestra hipótesis de trabajo de qué forma el filósofo ve en los personajes 'clownescos' de Beckett a los viejos duetistas del circo (la oposición
6. El crítico irlandés Seamus Deane, en su ya clásica comparación entre Joyce y Beckett, utilizará también el concepto de reducción, véase Deane (1985).

7. Ya Maurice Nadeau, a comienzos de la década del setenta, denominaba impugnación lo que Badiou llama "bloqueo": “La impugnación del lenguaje viene acompañada de una impugnación de la obra. Condenados a hablar, los héroes de Beckett se dedican a negar lo que acaban de afirmar, a decir al mismo tiempo sí y no. Si hay que hablar, al menos que sea para no decir nada", en Nadeau (1971).

8. “La voz puede tener el perfume de la verdad", en Sollers (2013: 110).

9. Badiou señala la "coincidencia" entre dos textos teatrales de Beckett y de Sartre emparentados por el uso del grabador. Recordemos que con una diferencia de pocos meses se estrenan en París: en septiembre de 1959 Les séquestrés de Altona de Jean Paul Sartre (Théâtre Récamier) y en marzo de 1960 La dernière bande de Samuel Beckett (Théâtre de la Renaissance). 10. Para un análisis, desde una visión latinoamericana, de Celan y su Anábasis con fuerte respaldo teórico filosófico y resonancias badiousianas, véase Olarte (2009). 
11. Hensel sostiene "El payaso es la clave para quien acude al teatro sin esperar encontrar en el escenario una cátedra de filosofía (continúa en página 23)

12. Para una interpretación sobre este "descenso al grotesco", véase Margarit (2003: 114). 13. Geneviève Serreau incluyó la dramaturgia de Beckett dentro de la etiqueta de Nouveau Théâtre. Por otra parte, así refiere las marcaciones cómicas de la actuación de En attendant Godot en su función de estreno (continúa en página 24)

14. Nos interesa la concepción y estudio de lo inarticulado (o voz confusa) que postula Giorgio Agamben en su texto "Pascoli y el pensamiento de la voz": "Los gramáticos antiguos comenzaban sus tratados desde la voz (foné). La voz, como puro sonido natural, no entra sin embargo en la gramática. (continúa en página 24)

15. Según el crítico Hugh Kenner, Beckett es un "comediante estoico" en una línea que continúa a Flaubert y a Joyce, donde 'estoico' se define como aquel que, sin pánico ni indiferencia, acuerda en que "el abanico de posibilidades" expresivas es grande, pero ya está cerrado. Beckett es, para Kenner, el comediante más extremo del impasse. (continúa en página 24)

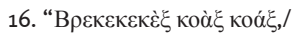

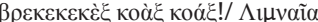

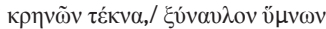

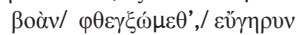

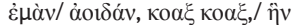

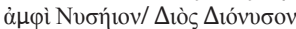

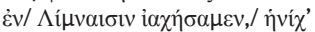

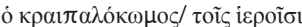

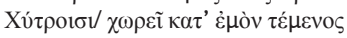

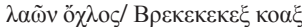

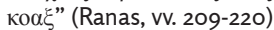

17. Según constató Segal, Aristófanes y su uso de la ironía es un fuerte "subtext" en similaridades manifiestas entre En attendant Godot de Beckett y Birds; véase Segal (1994).

18. Para una lectura sobre la importancia de Watt de Beckett en Badiou véase Rabaté (2005: 87-108): "Badiou takes Watt as a sounding board when he wishes to explain how Beckett has moved from a Cartesian universe (so dominant in Murphy) (continúa en página 25)

19. Condiciones de Badiou se cierra con un ensayo sobre Beckett donde profundiza las características peculiarísimas de los incidentes en Watt: “¿Qué son estos incidentes? Entre los más subrayados, citamos la visita de un afinador y de su hijo, o la colocación ante la puerta de marmitas con desechos destinados (continúa en página 25) entre el augusto y el payaso blanco $)^{11}$ donde ya no importa la intriga, la situación o el desenlace sino la mera confrontación ilimitada de "figuras extremas de la dualidad". En Citrouilles/Ranas - a modo de dispositivo-, pensamos en la puja entre Claudel y Brecht sobre el modelo aristofánico entre Esquilo y Eurípides, y rescatamos como esencial para nuestro estudio la postulación final de Badiou: en todo el siglo XX no hay otro autor tan descarnadamente "cómico" 12 como Samuel Beckett: ${ }^{13}$

Desde este punto de vista, Beckett está indiscutiblemente -y es el único gran escritor de este siglo que lo está- en una tradición sin par del teatro cómico: duetistas diferenciados, trajes desfasados (falsamente 'nobles', sombreros hongo, etc.), serie de números antes que desarrollo de una intriga, trivialidades, injurias y escatología, parodia del lenguaje culto, en especial del lenguaje filosófico, indiferencia con respecto a toda verosimilitud y, sobre todo, obstinación de los personajes en perseverar en su ser, en defender contra viento y marea un principio de deseo, una fuerza vital, que las circunstancias parecen transformar, en todo momento, en ilegítima e imposible. La minusvalía no es una metáfora patética de la condición humana. El teatro cómico está repleto de ciegos libidinosos, de ancianos impotentes que se empecinan en dejarse llevar por sus pasiones, de sirvientesesclavos acribillados de golpes, pero triunfantes, de jóvenes estúpidos, de cojos megalómanos...(...) Hay que interpretar a Beckett con el más intenso humorismo, en la variedad constante de los tipos teatrales heredados, y solamente entonces es cuando se ve surgir lo que de hecho es la verdadera razón de lo cómico: no un símbolo, tampoco una metafísica disfrazada, mucho menos un escarnio, sino un amor poderoso por la obstinación humana, por el infatigable deseo, por la humanidad reducida a su malicia y a su terquedad. Los personajes de Beckett son esos anónimos del quehacer humano que lo cómico vuelve a la vez intercambiable e irremplazable. (Badiou 2007:63-64)

Por su parte, no podemos soslayar que para Badiou la novela vanguardista y experimental Watt (1953) de Beckett es una indagación crucial acerca del acontecimiento y tiene particular interés en relación a nuestro trabajo sobre "el episodio del croar"14.

La "novela irlandesa" Watt escrita en inglés por Beckett ${ }^{15}$ en su estadía en Rousillon durante la guerra -entre febrero del 1941 y diciembre 1944-, en seis cuadernos que devinieron en cuatro capítulos y una addenda, implica un hito a efectos de la hipótesis que barajamos en este artículo por su clara referencia a uno los pasajes más celebres

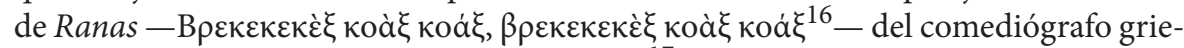
go Aristófanes, y de la historia de la literatura; ${ }^{17}$

Watt es un intérprete, un hermeneuta. Incluso la hipótesis del sinsentido está presa de una voluntad encarnizada de dar sentido y aún más de conectar ese sentido a un sentido original, un sentido perdido y redescubierto. (Badiou 2007: 37)

En la novela, Watt encarna al héroe del error y la falibilidad del lenguaje, "por no hablar de la tortura inducida por el imperativo del habla" (Badiou 2007:37); Watt es el héroe de la repetición y de la variabilidad de las palabras hasta el non sense, el desvarío o la "convulsión fónica": es la mostración del lenguaje en su versión centrífuga. ${ }^{18}$ En su "quête de signification" cada suceso, evento, episodio o momento serán motivos de incidentes "brillantes de claridad formal y con contenido impenetrable" (Badiou 2012: $331)^{19}$ que llevará a su lenguaje a un deterioro progresivo en la apuesta por la exploración minuciosa (y exhaustiva) del mundo y por la "cuantificación" de todo lo visible.

La novela "narra" el viaje de Watt, el primer clochard y vagabundo irlandés, en viaje a casa del señor Knott donde ingresa como ayuda doméstico (o valet, pensando en el 
Jantias aristofánico o en el Ahmed badiousiano) para sustituir a Arsene, que se retira al final del primer capítulo con un farragoso monólogo. El segundo capítulo - de interés porque incluye la "incrustación" sonora y secuencial del canto de las ranasejemplifica los denodados esfuerzos de Watt por 'dar sentido' a su trabajo en la casa despistado por episodios que le producen una extrañeza que acrecienta su propia y metafísica singularidad.

Si el Coro de Ranas aristofánico, traspuesto por Badiou en Coro de Calabazas, implica, visto desde la perspectiva beckettiana, un episodio mayúsculo de humorismo filosófico, la retórica de la ironía presente en las ranas ditirámbicas que se "muestran" sólo a través de sus onomatopeyas se reactiva en Watt como novela "anti-lógica” en el escandido croar $^{20}$ - hipercodificado- de las tres ranas y sus sonoridades cifradas, ${ }^{21}$ parodia y pastiche acaso de los sistemas lógicos y de las oposiciones binarias o racionalistas. En un juego sonoro Beckett escande el armonioso croar vocálico a modo de escritura en clave que estaría respondiendo a la célebre secuencia aritmética de Fibonacci (8-5-3). ${ }^{22}$ El grafismo juega con la disposición —no sólo espacial sino también sonora- de dieciséis tercetos de octosilábicos pies (si lo pensamos en clave métrica) o bien - acorde a la serialidad musical, que muchos críticos han reconocido- en términos de "octavas":

Watt remembered a distant summer night, in a no less distant land, and Watt young and well lying all alone stone sober in the ditch, wondering if it was the time and the place and the loved one already, and the three frogs croaking Krak!, Krek! and Krik!, at one, nine, seventeen, twenty-five, etc., and at one, six, eleven, sixteen, etc., and at one, four, seven, ten, etc., respectively, and how he heard

$$
\begin{aligned}
& \text { Krak! - - - - - - } \\
& \text { Krek! - - - Krek! - - } \\
& \text { Krik! - Krik! — Krik! - } \\
& \text { Krak! - - - - - - } \\
& \text { - - Krek! - - - Krek! } \\
& \text { — Krik! - Krik! — Krik! } \\
& \text { Krak! - - - - - - } \\
& \text { - - - Krek! - - - } \\
& \text { - - Krik! - Krik! - - } \\
& \text { Krak! - - - - - - } \\
& \text { - Krek! - - - Krek! - } \\
& \text { Krik! — Krik! — Krik! — } \\
& \text { Krak! - - - - - - } \\
& \text { - - - Krek! - - - - } \\
& \text { — Krik! — Krik! — Krik! } \\
& \text { Krak! - - - - - - } \\
& \text { Krek! - - - Krek! - - } \\
& \text { — Krik! — Krik! — - } \\
& \text { Krak! - - - - - - } \\
& \text { - - Krek! - - - Krek! } \\
& \text { Krik! — Krik! - Krik! - } \\
& \text { Krak! - - - - - - } \\
& \text { - - - Krek! - - - } \\
& \text { —Krik! — Krik! — Krik! }
\end{aligned}
$$

20. Alfonso Reyes sostiene y enumera: "La imitación poética de las aves tiene un bello antecedente aristofánico que todos conocen. A veces, los traductores traducen también a su modo las onomatopeyas de Aristófanes. La abubilla, dice: “Epopoi, epopoi. Epopo, popo, popo, popo, popoi io, io. Tío, tío, tío, tío, tío, tío tío, tío. Troito, troito, toio brix. Torotorotorotorotix. Kiccabau, Kiccabau. Torotorotorotorolililiy". El fenicóptero: "Torotix, tototix". El coro: "Po, po,po,po,po, po. $\mathrm{Ti}$, ti, ti, ti,ti,ti,ti. Nash oía cantar al ruiseñor: "Cuekoo, jug-jug, pu-we, tuwittawoo" $Y$ un autor que oculta su nombre oía repetir incansablemente a una pareja de pinzones:

"Bosuet, Bourdaloue". En André Salmon, Saints de glace, el ruiseñor parece inspirado en Aristófanes, pero como contemporáneo que es, ignora la medida clásica y alarga fatigosamente su onomatopeya desde "Tiouo" hasta "tsipi", en no menos de veinticinco versos, en general impronunciables. La jitanjáfora poética ataca también, como la popular, la imitación de otros animales. Las ranas de Aristófanes cantan: "Brekekekex, coax, coax” y Curros Enríquez oía cantar: "Crocro" a su "sapo lloroso”, véase el artículo “Las jitanjáforas” (Reyes, 1952: 214-215).

21. Según pudimos relevar sobre la interpretación crítica de este pasaje en ningún caso aparece la referencia aristofánica: Keller analiza el episodio en clave psicoanalítica con un intento de retorno de Watt a la armonía y "a la madre" (Keller, 2002)

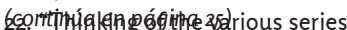
in his universe, Watt recalls the song of the three frogs (136-38), croaking Krak!, Krek! and Krik! at intervals, respectively, of eight, five and three, a Fibonacci series in which each interval is the sum of the two preceding ("Krok!" and "Kruk!” would be, presumably, two and one), beginning together but not croaking in unison again until after 120 bars (if the $3 / 4$ timing is taken from Krik!), or 360 beats.

This seems to be perfect harmony, a triumph of reason. Yet questions abide: does design imply cause, or pattern imply purpose? Reduced to the fundamental sounds of the frogs, these matters lie at the very heart of Watt, the title of which is also a fundamental question. The frog song exemplifies the serial elements in Watt's world, as anticipated in Proust (21-22): "The mortal microcosm cannot forgive the relative immortality of the macrocosm. The whisky bears a grudge against the decanter", en Ackerley. 


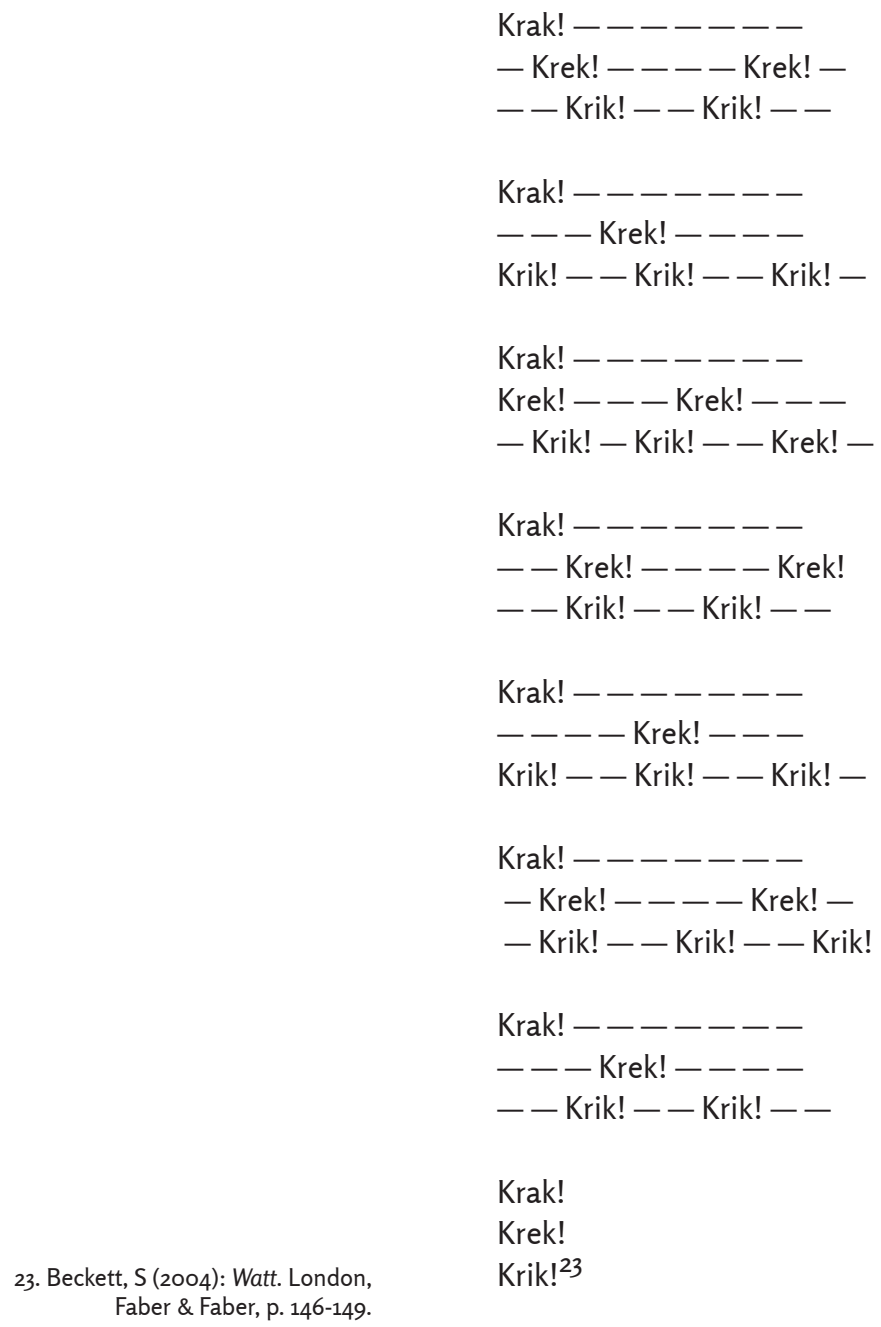

Para Badiou, entendido Watt como un hermeneuta, la tarea consiste - "mediante una interpretación bien conducida" - en hacer concertar el incidente al "universo establecido de las significaciones":

El hermeneuta tiene tres posibilidades: si supone que hay una significación del incidente, puede encontrarla o proponerse otra absolutamente diferente. Si supone que no hay significación, puede hacerle surgir una. Por supuesto, sólo la tercera hipótesis, que plantea que el incidente está desprovisto de toda significación, y que por consiguiente está separado del universo cerrado del sentido (la casa de Monsieur Knott), despierta duraderamente ('en un plazo más o menos largo') al pensamiento, le demanda un trabajo ('con más o menos dificultad'). Sin embargo, si no se trata más que de ello, si el intérprete es un donador de sentido, permanecemos prisioneros de la significación como ley, como imperativo. El intérprete no crea nada más que un ajuste del incidente a aquello de lo que en su origen se separaba: el universo establecido de las significaciones, la casa de Monsieur Knott. En Watt tenemos la posibilidad de que pase cualquier cosa, pero lo-que-pase, captado y reducido por el hermeneuta, no es preservado en su carácter de suplemento, o de mellamiento. (Badiou 2012: 331-332)

Pero, según postula Badiou, esa hermenéutica primaria que intenta acoplar el acontecimiento a la red de significaciones será reemplazada finalmente por Samuel Beckett por otro tipo de operación: la nominación que no busca ningún sentido y que se propone extraer - del vacío mismo "de lo que adviene" - un nombre inventado. La nominación 
no es ya más la cautiva de las significaciones; los textos "hablan" o croan por sí mismos: "Este ruido está fuera de lugar, está aislado en su claridad formal, es in-visible, mal visto" (Badiou 2012:331) A la instancia interpretativa le seguirá una poética nominal. Su única finalidad es 'fijar' el incidente.

Traspasada la teoría al coro aristofánico de Ranas en disputa con Dioniso, a ese "acontecimiento-ruido", esa nominación 'batracia' surge del "vacío de la lengua, como un mal decir adecuado a lo mal visto del ruido". Lo que resulta todavía más importante es que, una vez enunciado "el derrumbe lánguido" como nombre de lo repentino del ruido, como reto poético sobre lo "mal visto" (Mal vu, mal dit de Beckett publicado en francés en 1981), entonces, y sólo entonces, habrá "una luz de esperanza" (Badiou 2012:333)

La esperanza - que acaso permite luego de ese reto o duelo con las Ranas o con las Calabazas tanto a Dioniso como a Ahmed seguir "el viaje" - es del orden de la verdad como gracia de un "modesto comienzo". Acaso este significativo pasaje-incidente en Watt - como el agón entre Dioniso y las ranas o la intervención del coro de Calabazas- pueda ser leído como el obstáculo a vencer: "de ahí que el sujeto se des-clausure de su encierro y entre en el peligro de lo Otro, de sus ocurrencias, de sus figuras" (Badiou 2012: 334).

\section{Notas}

4 Sobre las cuestiones referidas al "poema latente" y a la prosa como textualidad genérica Artus-Bouvet así lo expone: "La prose désignerait en ce sens la capacité générique du poème, qui lui permet d'accueillir à la fois une duplicité énonciative, la motivation d'un récit et de ses simulacres, l'organisation d'un doute méthodique, et la profondeur d'une réflexivité de type métadiscoursif. La prose constitue alors la solution 'générique' de la contradiction initialement formulée par Mallarmé: le poème, on s'en souvient, exigeait le théâtre tout en y étant réputé 'non possible'. Or l'impossibilité théâtrale s'avère dans la disparition des nymphes: l'oeuvre, dès lors, n'a plus lieu qu'en tant qu'un monologue solitaire, ou la vocalité vivante de la scène fait place à la scripturalité du poème. Mais ce monologue, il faut y insister, continue en tant que tel d'exiger le théâtre: cette exigence, qui, sur le plan thématique, se réalise dans la parole nostalgique et douteuse, quoique jubilatoire, du faune, est identiquement une exigence générique. Elle a conduit Mallarmé à l'invention d'une 'prose' spécifique, dont la fonction est d'ouvrir le poème à des potentialités génériques non restrictivement poétiques", en Artous-Bouvet (2011: 9). (Viene de página 18.)

11 Hensel sostiene "El payaso es la clave para quien acude al teatro sin esperar encontrar en el escenario una cátedra de filosofía; a quienes, si van a ver a Beckett, esperan unos payasos en la escena. Sin duda Esperando a Godot es una pieza religiosa, aunque no cristiana: incesantemente formula preguntas sobre el sentido del ser y obstinadamente se niega a contestarlas", en Hensel (1972: 45). Sobre este mismo tópico, pero con otra lectura Giraudon sostiene: "Hay en Beckett una exploración teatral de ciertas posibilidades del circo: los monstruos, en primer término, y nos vuelve a la memoria Tod Browning, el creador de Drácula, en su película de 1932, interpretada por los pupilos de Barnum: La fiesta de los monstruos. Enseguida, los payasos. Una obra de Beckett es un drama shakesperiano reducido a sus payasos. (...) No vamos a entrar en la disputa: ¿payasos o vagabundos? En realidad se trata de payasos vagabundizados, vale decir que, en este dúo (Pozzo y Lucky) el payaso blanco ha desaparecido", en Giraudon (1972: 26). (Viene de página 20.) 
13 Geneviève Serreau incluyó la dramaturgia de Beckett dentro de la etiqueta de Nouveau Théâtre. Por otra parte, así refiere las marcaciones cómicas de la actuación de En attendant Godot en su función de estreno: “Una indicación escénica enfrentó ese día a Pierre Latour, que hacía el papel de Estragón, con Blin y Beckett. Latour se negó de pronto a dejar que, al fin de la pieza, cayeran sus pantalones a los tobillos -hasta entonces habían estado sostenidos por la cuerda con que Didi y Gogo trataban en vano de ahorcarse. ‘¿Qué voy a parecer en calzoncillos? —protestaba-. Pase aún que se rían de mí; pero además van a echarse a reír en el momento que no hace falta'. Beckett y Blin no cedieron. Era preciso que el pantalón cayera. Con el fin de que fuese levantado. Con el fin de que este último gesto, ridícula pero resueltamente, fuera el comienzo aún de la misma espera, el recomenzar indefinido del mismo círculo, de esta misma vida que no termina nunca y que, de no comenzar, no acabaría. Al día siguiente el pantalón cayó. Fue uno de los pocos momentos de la representación en que nadie sintió ganas de reír”, en Serreau (1967: 86). (Viene de página 20.)

14 Nos interesa la concepción y estudio de lo inarticulado (o voz confusa) que postula Giorgio Agamben en su texto "Pascoli y el pensamiento de la voz": "Los gramáticos antiguos comenzaban sus tratados desde la voz (foné). La voz, como puro sonido natural, no entra sin embargo en la gramática. Esta comienza, en efecto, con la distinción de la "voz confusa" de los animales (foné synkechyméne ho agrámmatos); los latinos traducían vox illiterata, quae litteris comprehendi non potest, (voz no escribible es la que no puede ser comprendida con letras) que no se puede escribir, como el equorum hinnitus (el relincho de los caballos) y la rabis canum (la rabia de los perros) de la voz humana escribible (engrámmatos) y articulada. Una clasificación más sutil, de origen estoico, distingue sin embargo la voz de modo más difuminado. Se debe saber -se lee en la Tékne grammatiké de Dionisio Tracio- que algunas de las voces son articuladas y escribibles (engrámmatoí), como las nuestras otras inarticuladas y no escribibles, como el crepitar del fuego y el fragor de las piedras o de la madera; otras inarticuladas y sin embargo escribibles, como las imitaciones de los animales irracionales, como el brekekéks y el koí; estas voces son inarticuladas porque no sabemos qué significan, pero no engrámatoi (escribibles), porque las podemos escribir. Detengámonos en estas voces inarticuladas y sin embargo "gramaticalizadas", en estos brekekéks y koí tan parecidos a las onomatopeyas pascolianas, y preguntémonos: ¿qué les sucedió a las confusas voces animales para haberse vuelto engrámmatos (escribibles), para haber sido encerradas en letras? Al entrar en los grammata (letras), al escribirse, se diferencian de la voz de la naturaleza, inarticulada e inscribible, para mostrase, en las letras, como un puro querer-decir, cuyo significado resulta desconocido (muy parecidos, en esto a la glosolalia y al vocabulum emortuum (palabra muerta) de Agustín). El único criterio que permite distinguirla de la voz inarticulada es, en efecto, que "no sabemos qué significa". El gramma, la letra, es, entonces, la cifra, en sí no significante, de una intención de significado que se cumplirá en el lenguaje articulado; el brekekéks, el koí y las otras imitaciones de las voces animales captan la voz de la naturaleza en el punto en el que emergen del mar infinito del mero sonido pero cuando no son aún lenguaje significante", en Agamben (2016: 130-132). (Viene de página 20.)

15 Según el crítico Hugh Kenner, Beckett es un “comediante estoico” en una línea que continúa a Flaubert y a Joyce, donde 'estoico' se define como aquel que, sin pánico ni indiferencia, acuerda en que "el abanico de posibilidades" expresivas es grande, pero ya está cerrado. Beckett es, para Kenner, el comediante más extremo del impasse. Impasse implica "no tener mucho que decir y ninguna razón para decirlo". Sobre Watt escribe: "Mientras más leemos Watt, más transige y se contradice la seria determinación de recobrar y registrar todo lo que es cognoscible de este espectral personaje; pues se vuelve cada vez más palpable que el libro es una composición ritualista, redundante, compulsiva, y el estilo enciclopédico y despreocupado ensaya de manera cada vez más evidente no hechos sino posibilidades, no evidencias sino especulación", en Kenner (2011: 117). (Viene de página 20.) 
18 Para una lectura sobre la importancia de Watt de Beckett en Badiou véase Rabaté (2005: 87-108): "Badiou takes Watt as a sounding board when he wishes to explain how Beckett has moved from a Cartesian universe (so dominant in Murphy) to a world in which the principle of the absurd does not rule out calculation but on the contrary starkly opposes the infinity of serial proliferation to the unique occurrence of an incomprehensible event In a crucial chapter entitled "The event and its name," Badiou sets the foundations for what is, according to him, Beckett's aim, the investigation of the minimal condition of freedom. This entails examining Watt, a novel in which "the prose oscillates between the grasp of an indifferent being and the torture of a reflection without any effect". (Viene de página 20.)

19 Condiciones de Badiou se cierra con un ensayo sobre Beckett donde profundiza las características peculiarísimas de los incidentes en Watt: “¿Qué son estos incidentes? Entre los más subrayados, citamos la visita de un afinador y de su hijo, o la colocación ante la puerta de marmitas con desechos destinados a perros cuya proveniencia es en sí mismo una pregunta 'impenetrable'. Lo que solicita al pensamiento es la contradicción entre la brillantez formal del incidente, su aislamiento, su estatus de excepción, y la opacidad de su contenido. Watt se desvive haciendo hipótesis sobre dicho contenido, es entonces verdaderamente cuando su pensamiento se despierta: No es aquí cuestión de un cogito bajo la coacción torturante de la voz, sino de cálculos y computaciones destinados a llevar al contenido de los incidentes a la altura de lo brillante de su forma. En Watt hay sin embargo un límite a esta investigación, limite que Beckett no franqueará sino más tarde: las hipótesis sobre los incidentes quedaban cautivas de una problemática de la significación" (Badiou, 2012: 331). (Viene de página 20.)

21 Según pudimos relevar sobre la interpretación crítica de este pasaje en ningún caso aparece la referencia aristofánica: Keller analiza el episodio en clave psicoanalítica con un intento de retorno de Watt a la armonía y "a la madre" (Keller, 2002); Kenner trabaja las nociones de falta de sincronicidad y de simultaneidad salvo en una única instancia dado que "each frog attends only to its private schedule of croaks" (Kenner, 1973: 86); Banfield observa en la forma fuertemente matemática del canto a modo de conteo, bajo un gesto acaso paródico de Beckett en tono a exhaustivas categorización y clasificación muy usual en Beckett (Banfield, 2012: 11-25); Deleuze sostiene que "En Watt, las tres ranas entremezclan sus canciones cada una con su cadencia propia (Krak!, Krek! y Krik!). Las imágenes-ritornellos corren a través de los libros de Beckett" (Deleuze, 1996: 6); Dennis trabaja con la idea desesperada de Watt de encontrar un "orden: "Play with sound and rhythm distracts from narrative chronology, frustrates intelligibility, and mocks rationalism's fondness for series and formulae. Exhaustive lists and permutations create patterns of sounds that establish an 'order' in the text that is other than semantics can occur via graphic representations of sound that resemble musical notation, as in a well-known passage consisting of frog noises:

Krak! - - - - - -

Krek! - - - Krek! - -

Krik! - - Krik! - - Krik! -

Nearly two pages of text are devoted to frog sounds followed by beats of rest. This interruption (or eruption) comes as Watt remembers 'a distant summer night, in a no less distant land, and Watt young and well lying all alone stone sober in the ditch, wondering if it was the time and the place and the loved one already, and the frogs croaking at one, nine, seventeen, twenty-five, etc.' (136). Watt's memory of the ardor he felt (for the fishwoman, Mrs. Gorman) overwhelms conceptual and discursive categories. Not only does the text give way to sound and rhythm -animal noises associated with sexual desire- but Watt, given his excessively rational temperament, imposes on the frogs' mating calls the regularity", en Dennis (2005: 4); Less lee el episodio, crucial para Watt, en clave tanto "existencial" como musical: "The next musical encounter -the Frog Song-appears much more detached from Watt's experience, firstly because it is not an actual event but 
a recollected one, and secondly because Watt seems to be aware only of the principle of order which underpins it. It is also worthy of note that whereas some composed music is given for the Threne (even if it is relegated to the Addenda) the Frog Song is given as a mere pattern of words. It seems significant in this connection that Beckett himself should not actually use the term 'song' to describe the incident nor ever state that the frogs actually 'sing'. I have retained the designation Frog Song in order to demonstrate later that there is an element of specially musical significance in the passage -indeed the Frog Song, in a musical context, is crucial to the process of Watt's 'untuning'. But it is obvious that the words of the frogs, only minimally musical in themselves, are not subjected to any type of musical dynamic by way of barring or phrasing, and that the silent beats are represented by dashes rather than by rests. Insofar as it appears as a musical experience at all, the Frog Song unquestionably takes place outside of Watt. There is, surely symptomatically, no linking phrase in the manner of 'he heard'. The man and the song are not related, merely juxtaposed", en http://www.english.fsu.edu/jobs/numog/ NumgLees.htm. (Viene de página 21.) 


\section{Q Bibliografía}

» Ackerley, C. (s/f). “Samuel Beckett and Mathematics”, http://www.uca.edu.ar/ uca/common/grupo17/files/mathem.pdf

»Agamben, G. (2016). El final del poema. Estudios de poética y literatura. Buenos Aires: Adriana Hidalgo, 130-132

"Aristofanes (2011). Ranas (edición bilingüe). Buenos Aires: Editorial de la Facultad de Filosofía y Letras Universidad de Buenos Aires. Edición revisada con traducción, introducción, notas y apéndices por Diana Frenkel, Ma. José Coscolla, Pablo Cavallero, Claudia Fernández, Ezequiel Rivas, Patricia Fernández y Silvina Schvartz.

» Artous-Bouvet , G. (2011). “Le Silence du faune: note sur L'après-midi d'un faune" Loxias, 33, 9.

»Badiou, A. (1996). Les Citrouilles. Paris: Actes Sud.

"Badiou, A. (2007). Beckett. El infatigable deseo. Madrid: Arena Libros.

»Badiou, A. (2010). La tétralogie d'Ahmed. Ahmed le subtil. Ahmed philosophe. Ahmed se fäche. Les Citrouilles. Paris: Actes Sud.

» Badiou, A. (2012). Condiciones. Buenos Aires: Siglo Veintiuno.

»Badiou, A. (2014). Elogio del teatro. Buenos Aires: Nueva Visión.

» Banfield, A. (2012). “Il pesimismo di Proust: un antídoto per Beckett” en CoSMo. Comparative Studies in Modernism, Vol 1, 11-25.

» Beckett, S. (1966). El innombrable. Barcelona: Lumen.

》 Beckett, S. (2004). Watt. Londres: Faber \& Faber.

"Casanova, P. (2001). “James Joyce y Samuel Beckett, o la autonomía”. La República mundial de las Letras. Barcelona: Anagrama.

"Deane, S. (1985). Celtic Revivals. London: Faber and Faber.

»Deleuze, G. (1996). “El agotado”, en Confines № 3. Buenos Aires: Lamarca/ Eudeba.

»Dennis, A. (2005). "Glitches in Logic in Beckett's Watt: Toward a Sensory Poetics". Journal of Modern Literature vol. 38, № 2.

» Giraudon, R. (1972). Demencia y muerte del teatro. México: Extemporáneos, p. 26

»Hensel, G. (1972). Samuel Beckett. México: FCE.

"Keller, J. R. (2002). Samuel Beckett and the primacy of love. NYC: Manchester University Press.

» Kenner, H. (1973). Samuel Beckett. A critical study. Berkeley: University of California Press.

» Kenner, H. (2011). Flaubert, Joyce y Beckett. Los comediantes estoicos. México: FCE.

»Lecercle, J.-J. (2010). Badiou and Deleuze. Edinburgh: Edinburgh University Press.

» Margarit, L. (2003). Samuel Beckett. Las huellas en el vacío. Buenos Aires: Atuel/ La avispa. 
» Nadeau, M. (1971). La novela francesa después de la guerra. Caracas: Tiempo Nuevo.

»Olarte, C. (2009). Paul Celan: meridiano de resistencia. Caracas: Fundación Editorial El perro y la rana.

»Pellettieri, O. (ed.) (2000). "La temática del sujeto en el teatro francés contemporáneo", Itinerarios del teatro latinoamericano. Buenos Aires: Galerna, 265-271.

»Rabaté, J. M. 2005. "U n b r e a k a b I e S 's From Beckett and Badiou to the Bitter End of Affirmative Ethics", en Riera, G. (ed.). Alain Badiou. Philosophy and its conditions. New York: State University of New York Press, 87-108.

"Saba Akim, M. (2013). "Absurdism as a Tendency in Theatre: Ranging from Aristophanes to Beckett and Pinter”. Lapis Lazuli: An International Literary Journal (LLILJ), Vol 3, № 2.

"Segal, E. (1994). “Aristophanes and Beckett” en Bierl et al. (eds), Orchestra, Springer Fachmeiden Wiesbaden

»Serreau, G. (1967). Historia del Nouveau Théâtre. México: Siglo XXI.

» Sollers, P. (2013). "Beckett conmovedor", en Discurso perfecto. Ensayo sobre literatura y arte Buenos Aires: El cuenco de plata. 